\title{
Center Manifold Reduction and Perturbation Method in a Delayed Model with a Mound-Shaped Cobb-Douglas Production Function
}

\author{
Massimiliano Ferrara, ${ }^{1}$ Luca Guerrini, ${ }^{2}$ and Giovanni Molica Bisci ${ }^{3}$ \\ ${ }^{1}$ Department of Law and Economics, University Mediterranea of Reggio Calabria, Via dei Bianchi 2 (Palazzo Zani), \\ 89127 Reggio Calabria, Italy \\ ${ }^{2}$ Department of Management, Polytechnic University of Marche, Piazza Martelli 8, 60121 Ancona, Italy \\ ${ }^{3}$ Department of PAU, University Mediterranea of Reggio Calabria, Via Melissari 24, 89124 Reggio Calabria, Italy
}

Correspondence should be addressed to Luca Guerrini; luca.guerrini@univpm.it

Received 29 October 2013; Accepted 20 November 2013

Academic Editor: Carlo Bianca

Copyright (C) 2013 Massimiliano Ferrara et al. This is an open access article distributed under the Creative Commons Attribution License, which permits unrestricted use, distribution, and reproduction in any medium, provided the original work is properly cited.

\begin{abstract}
Matsumoto and Szidarovszky (2011) examined a delayed continuous-time growth model with a special mound-shaped production function and showed a Hopf bifurcation that occurs when time delay passes through a critical value. In this paper, by applying the center manifold theorem and the normal form theory, we obtain formulas for determining the direction of the Hopf bifurcation and the stability of bifurcating periodic solutions. Moreover, Lindstedt's perturbation method is used to calculate the bifurcated periodic solution, the direction of the bifurcation, and the stability of the periodic motion resulting from the bifurcation.
\end{abstract}

\section{Introduction}

In recent years, great attention has been paid to economic growth models with time delay. The reason is that, getting closer to the real world, there is always a delay between the time when information is obtained and the time when the decision is implemented. Different mathematical and computational frameworks have been proposed whose difficulty is strictly related to the phenomena of the system that has to be modeled. The inclusion of delay in these systems has illustrated more complicated and richer dynamics in terms of stability, bifurcation, periodic solutions, and so on. For examples, see Asea and Zak [1], Zak [2], Szydłowski [3], Szydłowski and Krawiec [4], Matsumoto and Szidarovszky [5], Matsumoto et al. [6], d'Albis et al. [7], Bambi et al. [8], Boucekkine et al. [9], Matsumoto and Szidarovszky [10], Ballestra et al. [11], Bianca and Guerrini [12], Bianca et al. [13], Guerrini and Sodini $[14,15]$, and Matsumoto and Szidarovszky [16]. However, in some of these papers the formulas for determining the properties of Hopf bifurcation were not derived.
This paper is concerned with the study of Hopf bifurcation of the model system with a fixed time delay presented in Matsumoto and Szidarovszky [5], where a continuous-time neoclassical growth model with time delay was developed similarly in spirit and functional form to Day's [17] discretetime model. Specifically, they have proposed the following delay differential equation:

$$
\dot{k}=-\alpha k+\beta k_{d}\left(1-k_{d}\right),
$$

where $k$ is the per capita per labor and $\alpha, \beta$ are positive parameters. In order to simplify the notation, we omit the indication of time dependence for variables and derivatives referred to as time $t$. As well, we use $k_{d}$ to indicate the state of the variable $k$ at time $t-\tau$, where $\tau$ represents the time delay inherent in the production process. According to Matsumoto and Szidarovszky [5], (1) has a unique positive steady state

$$
k_{*}=\frac{\beta-\alpha}{\beta},
$$


if $\beta>\alpha$. In case $\beta>3 \alpha$, this equilibrium is locally asymptotically stable for $\tau<\tau_{*}$ and unstable for $\tau>\tau_{*}$, where

$$
\tau_{*}=\frac{\cos ^{-1}(\alpha /(2 \alpha-\beta))}{\omega_{*}}, \quad \text { with } \omega_{*}=\sqrt{(\beta-\alpha)(\beta-3 \alpha)} .
$$

The change in stability will be accompanied by the birth of a limit cycle in a Hopf bifurcation. This limit cycle will start with zero amplitude and will grow as $\tau$ is further increased. Using the theory of normal form and center manifold (see [18]), we extend their analysis, providing formulas for determining the stability of the bifurcating periodic solutions and the direction of the Hopf bifurcation. Finally, even if the literature on economic models with delays is quite huge, we have noticed that the study of the type of Hopf bifurcation is really rare. Therefore, we have deepened this last point by using the perturbation method known as Lindstedt's expansion (see, e.g., $[19,20])$ and furnished a detailed analysis on approximation to the bifurcating periodic solutions.

\section{Direction and Stability of Bifurcating Periodic Solutions}

In this section, we study the direction, stability, and period of the bifurcating periodic solutions in (1) that are generated at the positive equilibrium when $\tau=\tau^{*}$. We let $i \omega_{*}$ be the corresponding purely imaginary root of the characteristic equation of the linearized equation of (1) at the positive equilibrium. The method we used is based on the normal form theory and the center manifold theorem introduced in Hassard et al. [18]. For notational convenience, let $\tau=\tau_{*}+\mu$, where $\mu \in \mathbb{R}$, so that $\mu=0$ is the Hopf bifurcation value for (1). First we use the transformation $x=k-k_{*}$, so that (1) becomes

$$
\dot{x}=-\alpha x+(2 \alpha-\beta) x_{d}-\beta x_{d}^{2} .
$$

Let $C=C\left(\left[-\tau_{*}, 0\right], \mathbb{R}\right)$ be the Banach space of continuous mappings from $\left[-\tau_{*}, 0\right]$ into $\mathbb{R}$ equipped with supremum norm. Let $x_{t}=x(t+\theta)$, for $\theta \in\left[-\tau_{*}, 0\right]$. Then, (4) can be written as

$$
\dot{x}(t)=L_{\mu}\left(x_{t}\right)+\mathscr{F}\left(\mu, x_{t}\right),
$$

where the linear operator $L_{\mu}$ and the function $\mathscr{F}$ are given by

$$
\begin{gathered}
L_{\mu}(\varphi)=-\alpha \varphi(0)+(2 \alpha-\beta) \varphi(-\tau), \\
\mathscr{F}(\mu, \varphi)=-\beta \varphi(-\tau)^{2},
\end{gathered}
$$

with $\varphi \in C$. By the Riesz representation theorem, there exists a bounded variation function $\eta(\theta, \mu), \theta \in\left[-\tau_{*}, 0\right]$, such that

$$
L_{\mu} \varphi=\int_{-\tau_{*}}^{0} d \eta(\theta, \mu) \varphi(\theta)
$$

where

$$
\eta(\theta, \mu)=-\alpha \delta(\theta)+(2 \alpha-\beta) \delta(\theta+\tau)
$$

with $\delta$ representing the Dirac delta function. Next, for $\varphi \in$ $C$, define

$$
\begin{gathered}
A(\mu)(\varphi)= \begin{cases}\frac{d \varphi(\theta)}{d \theta}, & \theta \in\left[-\tau_{*}, 0\right), \\
\int_{-\tau_{*}}^{0} d \eta(r, \mu) \varphi(r), & \theta=0,\end{cases} \\
R(\mu)(\varphi)= \begin{cases}0, & \theta \in\left[-\tau_{*}, 0\right), \\
\mathscr{F}(\mu, \varphi), & \theta=0 .\end{cases}
\end{gathered}
$$

As a result, (5) can be expressed as

$$
\dot{x}_{t}=A(\mu) x_{t}+R(\mu) x_{t} .
$$

For $\psi \in \widetilde{C}=C\left(\left[0, \tau_{*}\right], \mathbb{R}\right)$, the adjoint operator $A^{*}$ of $A$ is defined as

$$
A^{*}(\mu) \psi(r)= \begin{cases}-\frac{d \psi(r)}{d r}, & r \in\left(0, \tau_{*}\right], \\ \int_{-\tau_{*}}^{0} d \eta(\zeta, \mu) \psi(-\zeta), & r=0 .\end{cases}
$$

Let $q(\theta)$ (resp., $q^{*}(\theta)$ ) denote the eigenvector for $A(0)$ (resp., for $A^{*}(0)$ ) corresponding to $\tau_{*}$; namely, $A(0) q(\theta)=$ $i \omega_{*} q(\theta)$ (resp., $A^{*}(0) q^{*}(r)=-i \omega_{*} q^{*}(r)$ ). To construct the coordinates to describe the center manifold near the origin, we define an inner product as follows:

$$
\langle\psi, \varphi\rangle=\bar{\psi}(0) \varphi(0)-\int_{\theta=-\tau_{*}}^{0} \int_{\xi=0}^{\theta} \bar{\psi}(\xi-\theta) d \eta(\theta) \varphi(\xi) d \xi
$$

for $\varphi \in C$ and $\psi \in \widetilde{C}$, where $d \eta(\theta)=d \eta(\theta, 0)$ and $\bar{\psi}$ represents the complex conjugate operation of $\psi$. The vectors $q$ and $q^{*}$ can be normalized by the conditions $\left\langle q^{*}, q\right\rangle=1$ and $\left\langle q^{*}, \bar{q}\right\rangle=0$. A direct computation shows that

$$
\begin{aligned}
& q(\theta)=e^{i \omega_{*} \theta}, \quad \theta \in\left[-\tau_{*}, 0\right], \\
& q^{*}(r)=B e^{i \omega_{*} r}, \quad r \in\left[0, \tau_{*}\right],
\end{aligned}
$$

where

$$
B=\frac{1}{1+(2 \alpha-\beta) \tau_{*} e^{i \omega_{*} \tau_{*}}} .
$$

Let $z=\left\langle q^{*}, x_{t}\right\rangle$ and

$$
W(t, \theta)=x_{t}(\theta)-2 \operatorname{Re}\{z q(\theta)\} .
$$

On the center manifold $C_{0}, W(t, \theta)=W(z, \bar{z}, \theta)$, with

$$
W(z, \bar{z}, \theta)=W_{20}(\theta) \frac{z^{2}}{2}+W_{11}(\theta) z \bar{z}+W_{02}(\theta) \frac{\bar{z}^{2}}{2}+\cdots,
$$

where $z$ and $\bar{z}$ are local coordinates for $C_{0}$ in the direction of $q^{*}$ and $\bar{q}^{*}$, respectively. For any $x_{t} \in C_{0}$ solution of (10), we have

$$
\begin{aligned}
\dot{z} & =\left\langle q^{*}, \dot{x}_{t}\right\rangle=\left\langle q^{*}, A(\mu) x_{t}+R(\mu) x_{t}\right\rangle \\
& =i \omega_{*} z+\bar{q}^{*}(0) \mathscr{F}_{0}(z, \bar{z})=i \omega_{*} z+g(z, \bar{z}),
\end{aligned}
$$


where $\mathscr{F}_{0}(z, \bar{z})=\mathscr{F}\left(0, x_{t}\right)$ and $g(z, \bar{z})=\bar{B}_{\mathscr{F}_{0}}(z, \bar{z})$. Noting from (16) that

$$
x_{t}(\theta)=W(z, \bar{z}, \theta)+z q(\theta)+\bar{z} \bar{q}(\theta),
$$

it follows that

$$
\begin{aligned}
& g(z, \bar{z}) \\
&=-\beta \bar{B} e^{-2 i \omega_{*} \tau_{*}} z^{2}-2 \beta \bar{B} z \bar{z}-\beta \bar{B} e^{2 i \omega_{*} \tau_{*}} \bar{z}^{2} \\
&-\beta \bar{B}\left\{\left[2 W_{11}\left(-\tau_{*}\right) e^{-i \omega_{*} \tau_{*}}+W_{20}\left(-\tau_{*}\right) e^{i \omega_{*} \tau_{*}}\right] z^{2} \bar{z}\right. \\
&\left.+\left[2 W_{11}\left(-\tau_{*}\right) e^{i \omega_{*} \tau_{*}}+W_{02}\left(-\tau_{*}\right) e^{-i \omega_{*} \tau_{*}}\right] z \bar{z}^{2}\right\} .
\end{aligned}
$$

Expanding $g(z, \bar{z})$ in powers of $z$ and $\bar{z}$, that is,

$$
g(z, \bar{z})=g_{20} \frac{z^{2}}{2}+g_{11} z \bar{z}+g_{02} \frac{\bar{z}^{2}}{2}+g_{21} \frac{z^{2} \bar{z}}{2}+\cdots,
$$

and comparing the above coefficients with those in (20), we get

$$
\begin{gathered}
g_{20}=-2 \beta \bar{B} e^{-2 i \omega_{*} \tau_{*}}, \quad g_{11}=-2 \beta \bar{B} \\
g_{02}=-2 \beta \bar{B} e^{2 i \omega_{*} \tau_{*}} \\
g_{21}=-2 \bar{B} \beta\left[2 W_{11}\left(-\tau_{*}\right) e^{-i \omega_{*} \tau_{*}}+W_{20}\left(-\tau_{*}\right) e^{i \omega_{*} \tau_{*}}\right] .
\end{gathered}
$$

In order to compute $g_{21}$, we need to know $W_{20}(0), W_{20}\left(-\tau_{*}\right)$ and $W_{11}(0), W_{11}\left(-\tau_{*}\right)$ first. From (16), one has

$$
\begin{aligned}
\dot{W} & =\dot{x}_{t}-\dot{z} q-\dot{\bar{z}} \bar{q} \\
& = \begin{cases}A W-2 \operatorname{Re}\left\{\bar{B} \mathscr{F}_{0} q(\theta)\right\}, & \theta \in\left[-\tau_{*}, 0\right), \\
A W-2 \operatorname{Re}\left\{\bar{B} \mathscr{F}_{0}\right\}+\mathscr{F}_{0}, & \theta=0\end{cases} \\
& =A W+H(z, \bar{z}, \theta),
\end{aligned}
$$

where

$$
H(z, \bar{z}, \theta)=H_{20}(\theta) \frac{z^{2}}{2}+H_{11}(\theta) z \bar{z}+H_{02}(\theta) \frac{\bar{z}^{2}}{2}+\cdots
$$

Recalling (23), it follows that

$$
\begin{aligned}
H(z, \bar{z}, \theta)= & -2 \operatorname{Re}\left\{\bar{B} \mathscr{F}_{0} q(\theta)\right\}=-g q(\theta)-\bar{g} \bar{q}(\theta) \\
= & -\left(g_{20} \frac{z^{2}}{2}+g_{11} z \bar{z}+g_{02} \frac{\bar{z}^{2}}{2}+\cdots\right) q(\theta) \\
& -\left(\bar{g}_{20} \frac{\bar{z}^{2}}{2}+\bar{g}_{11} z \bar{z}+g_{02} \frac{z^{2}}{2}+\cdots\right) \bar{q}(\theta) .
\end{aligned}
$$

On the other hand,

$$
\begin{gathered}
\dot{W}_{20}(\theta)=2 i \omega_{*} W_{20}(\theta)-H_{20}(\theta), \\
A W_{11}(\theta)=-H_{11}(\theta) .
\end{gathered}
$$

A comparison of the coefficients of (24) and (25) gives

$$
\begin{aligned}
& H_{20}(\theta)=-g_{20} q(\theta)-\bar{g}_{02} \bar{q}(\theta), \\
& H_{11}(\theta)=-g_{11} q(\theta)-\bar{g}_{11} \bar{q}(\theta) .
\end{aligned}
$$

Thus, (26) becomes

$$
\dot{W}_{20}(\theta)=2 i \omega_{*} W_{20}(\theta)+g_{20} q(\theta)+\bar{g}_{02} \bar{q}(\theta),
$$

which is solved by

$$
W_{20}(\theta)=-\frac{g_{20}}{i \omega_{*}} e^{i \omega_{*} \theta}-\frac{\bar{g}_{02}}{3 i \omega_{*}} e^{-i \omega_{*} \theta}+E_{1} e^{2 i \omega_{*} \theta} .
$$

Similarly, from

$$
\dot{W}_{11}(\theta)=g_{11} q(\theta)+\bar{g}_{11} \bar{q}(\theta),
$$

we derive

$$
W_{11}(\theta)=\frac{g_{11}}{i \omega_{*}} e^{i \omega_{*} \theta}-\frac{\bar{g}_{11}}{i \omega_{0}} e^{-i \omega_{*} \theta}+E_{2}
$$

where $\left(E_{1}, E_{2}\right)$ is a constant vector. In order to compute $W_{20}$ and $W_{11}$, the constants $E_{1}$ and $E_{2}$ are needed. From (23), we have

$$
H(z, \bar{z}, 0)=-2 \operatorname{Re}\left\{\bar{B}_{\mathscr{F}_{0}} q(0)\right\}+\mathscr{F}_{0} .
$$

Thus,

$$
\begin{gathered}
H_{20}(0)=-g_{20}-\bar{g}_{20} \bar{B}-2 \beta e^{-2 i \omega_{*} \tau_{*}}, \\
H_{11}(0)=-g_{11}-\bar{g}_{11} \bar{B}-2 \beta .
\end{gathered}
$$

On the center manifold, we have $\dot{W}=W_{z} \dot{z}+W_{\bar{z}} \dot{\bar{z}}$. Replacing $W_{z}, W_{\bar{z}}$ and $\dot{z}, \dot{\bar{z}}$, we obtain a second expression for $\dot{W}$. A comparison of the coefficients of this equation with those in (23), for $\theta=0$, leads us to the following:

$$
\begin{gathered}
\left(A-2 i \omega_{*}\right) W_{20}(0)=-H_{20}(0), \\
A W_{11}(0)=-H_{11}(0) .
\end{gathered}
$$

Since

$$
\begin{aligned}
& A W_{20}(0)=-\alpha W_{20}(0)+(2 \alpha-\beta) W_{20}\left(-\tau_{*}\right), \\
& A W_{11}(0)=-\alpha W_{11}(0)+(2 \alpha-\beta) W_{11}\left(-\tau_{*}\right),
\end{aligned}
$$

from the previous analysis we arrive at

$$
\begin{gathered}
-\alpha W_{20}(0)+(2 \alpha-\beta) W_{20}\left(-\tau_{*}\right)-2 i \omega_{*} W_{20}(0) \\
=g_{20} q(0)+\bar{g}_{20} \bar{q}(0)+2 \beta e^{-2 i \omega_{*} \tau_{*}}, \\
-\alpha W_{11}(0)+(2 \alpha-\beta) W_{11}\left(-\tau_{*}\right)=g_{11} q(0)+\bar{g}_{11} \bar{q}(0)+2 \beta .
\end{gathered}
$$

Hence, $E_{1}$ and $E_{2}$ can be computed from (29) and (31) as $\theta=$ 0 , and we obtain

$$
E_{1}=\frac{F_{1}}{-\alpha+(2 \alpha-\beta) e^{-2 i \omega_{*} \tau_{*}}-2 i \omega_{*}},
$$


where

$$
\begin{aligned}
& F_{1}=\left(-\alpha-2 i \omega_{*}\right)\left(\frac{g_{20}}{i \omega_{*}}+\frac{\bar{g}_{02}}{3 i \omega_{*}}\right) \\
&+(2 \alpha-\beta)\left(\frac{g_{20}}{i \omega_{*}} e^{-i \omega_{*} \tau_{*}}+\frac{\bar{g}_{02}}{3 i \omega_{*}} e^{i \omega_{*} \tau_{*}}\right) \\
&+g_{20}+\bar{g}_{02}+2 \beta e^{-2 i \omega_{*} \tau_{*}}, \\
& E_{2}=\frac{F_{2}}{-\alpha+(2 \alpha-\beta)},
\end{aligned}
$$

where

$$
\begin{aligned}
F_{2}= & \alpha\left(\frac{g_{11}}{i \omega_{*}}-\frac{\bar{g}_{11}}{i \omega_{*}}\right)-(2 \alpha-\beta)\left(\frac{g_{11}}{i \omega_{*}} e^{i \omega_{*} \tau_{*}}-\frac{\bar{g}_{11}}{i \omega_{*}} e^{-i \omega_{*} \tau_{*}}\right) \\
& +g_{11}+\bar{g}_{11}+2 \beta .
\end{aligned}
$$

Based on the above analysis, all $g_{i j}$ have been obtained. Consequently, we can compute the following quantities:

$$
\begin{gathered}
C_{1}(0)=\frac{i}{2 \omega_{*}}\left(g_{11} g_{20}-2\left|g_{11}\right|^{2}-\frac{\left|g_{02}\right|^{2}}{3}\right)+\frac{g_{21}}{2}, \\
\mu_{2}=-\frac{\operatorname{Re}\left[C_{1}(0)\right]}{\operatorname{Re}\left\{\lambda^{\prime}\left(\tau_{*}\right)\right\}}, \quad \beta_{2}=2 \operatorname{Re}\left[C_{1}(0)\right] \\
T_{2}=-\frac{\operatorname{Im}\left[C_{1}(0)\right]+\mu_{2} \operatorname{Im}\left[\lambda^{\prime}\left(\tau_{*}\right)\right]}{\omega_{*}},
\end{gathered}
$$

which determine the quantities of bifurcating periodic solutions in the center manifold at the critical value. We will summarize it in the following result.

Theorem 1. Let $C_{1}(0), \mu_{2}, \beta_{2}$, and $T_{2}$ be defined in (40).

(i) The bifurcating periodic solution is supercritical bifurcating as $\operatorname{Re}\left[C_{1}(0]\right)>0$, and it is subcritical bifurcating as $\operatorname{Re}\left[C_{1}(0]\right)<0$.

(ii) The bifurcating periodic solutions are stable if $\operatorname{Re}\left[C_{1}(0]\right)<0$ and unstable if $\operatorname{Re}\left[C_{1}(0]\right)>0$.

(iii) As $\tau$ increases, the period of bifurcating periodic solutions increases if $T_{2}>0$, while it decreases, if $T_{2}<0$.

\section{Lindstedt's Method}

In the previous section, the direction and stability of the Hopf bifurcation were investigated by using the normal form theory and the center manifold theorem as in Hassard et al. [18]. Specifically, the delay differential equation of our model was converted into an operator equation on a Banach space of infinite dimension and then simplified into a onedimensional ordinary differential equations on the center manifold. Now we will use a different approach to investigate periodic solutions of (4), namely, of (1), which consists in applying Lindstedt's perturbation method (see, e.g., $[19,20]$ ). To this end, we start stretching time with the transformation

$$
s=\omega t
$$

so that solutions of (4) which are $2 \pi / \omega$ periodic in $t$ become $2 \pi$ periodic in $s$. This change of variables results in the following form of (4):

$$
\omega \frac{d x(s)}{d s}=a_{0} x(s)+a_{1} x(s-\omega \tau)+a_{2} x(s-\omega \tau)^{2},
$$

where the terms $a_{0}, a_{1}$, and $a_{2}$ are given by

$$
a_{0}=-\alpha<0, \quad a_{1}=2 \alpha-\beta<0, \quad a_{2}=-\beta<0 .
$$

The idea is now to expand the solution of (42) in a power series in a suitable smallness parameter $\varepsilon$, that is,

$$
x(s)=x_{0}(s) \varepsilon+x_{1}(s) \varepsilon^{2}+x_{2}(s) \varepsilon^{3}+\cdots,
$$

and to solve for the unknown functions $x_{j}(s)$ recursively. In this context, the definition of the $x_{j}(s)(j=0,1,2, \ldots)$ is clear. As already mentioned, $\varepsilon$ represents a small quantity so that we can expand the frequency $\omega$ and the delay $\tau$ in powers of $\varepsilon$ according to

$$
\begin{gathered}
\omega=\omega(\varepsilon)=\omega_{0}+\omega_{1} \varepsilon+\omega_{2} \varepsilon^{2}+\cdots, \\
\tau=\tau(\varepsilon)=\tau_{0}+\tau_{1} \varepsilon+\tau_{2} \varepsilon^{2}+\cdots,
\end{gathered}
$$

where we have set

$$
\tau_{0}=\tau_{*}, \quad \omega_{0}=\omega_{*} .
$$

In addition, we also have to consider a corresponding expansion of the time delayed term $x(s-\omega \tau)$, which is achieved by

$$
\begin{aligned}
x(s-\omega \tau)= & x_{0}(s-\omega \tau) \varepsilon+x_{1}(s-\omega \tau) \varepsilon^{2} \\
& +x_{2}(s-\omega \tau) \varepsilon^{3}+\cdots
\end{aligned}
$$

where $x_{j}(s-\omega \tau)$ stands for

$$
\begin{aligned}
x_{j}(s & -\omega \tau) \\
= & x_{j}\left(s-\omega_{0} \tau_{0}\right)-x_{j}^{\prime}\left(s-\omega_{0} \tau_{0}\right) \\
& \times\left[\left(\omega_{1} \tau_{0}+\omega_{0} \tau_{1}\right) \varepsilon+\left(\omega_{2} \tau_{0}+\omega_{1} \tau_{1}+\omega_{0} \tau_{2}\right) \varepsilon^{2}+\cdots\right] \\
& +\frac{1}{2} x_{j}^{\prime \prime}\left(s-\omega_{0} \tau_{0}\right)\left[\left(\omega_{1} \tau_{0}+\omega_{0} \tau_{1}\right) \varepsilon+\cdots\right]^{2}-\cdots
\end{aligned}
$$

with primes representing differentiation with respect to $s$. Applying the expansions for $x(s)$ and $x(s-\omega \tau)$ to $(42)$ and collecting terms for the distinct orders of $\varepsilon$, we get the following three equations:

$$
\begin{aligned}
O(\varepsilon): \omega_{0} \frac{d x_{0}(s)}{d s}=a_{0} x_{0}\left(s-\omega_{0} \tau_{0}\right)+a_{1} x_{0}\left(s-\omega_{0} \tau_{0}\right), \\
O\left(\varepsilon^{2}\right): \omega_{0} \frac{d x_{1}(s)}{d s}-a_{0} x_{1}(s)-a_{1} x_{1}\left(s-\omega_{0} \tau_{0}\right) \\
=-\omega_{1} \frac{d x_{0}(s)}{d s}-a_{1} x_{0}^{\prime}\left(s-\omega_{0} \tau_{0}\right)\left(\omega_{1} \tau_{0}+\omega_{0} \tau_{1}\right) \\
+x_{0}^{2}(s)+a_{2} x_{0}^{2}\left(s-\omega_{0} \tau_{0}\right),
\end{aligned}
$$




$$
\begin{aligned}
O\left(\varepsilon^{3}\right): & \omega_{0} \frac{d x_{2}(s)}{d s}-a_{0} x_{2}(s)-a_{1} x_{2}\left(s-\omega_{0} \tau_{0}\right) \\
= & -\omega_{2} \frac{d x_{0}(s)}{d s}-a_{1} x_{0}^{\prime}\left(s-\omega_{0} \tau_{0}\right)\left(\omega_{2} \tau_{0}+\omega_{1} \tau_{1}+\omega_{0} \tau_{2}\right) \\
& +2 a_{2} x_{0}\left(s-\omega_{0} \tau_{0}\right) x_{1}\left(s-\omega_{0} \tau_{0}\right)-\omega_{2} \frac{d x_{0}(s)}{d s} \\
& -a_{1} x_{0}^{\prime}\left(s-\omega_{0} \tau_{0}\right)\left(\omega_{2} \tau_{0}+\omega_{1} \tau_{1}+\omega_{0} \tau_{2}\right) \\
& -2 a_{2} x_{0}\left(s-\omega_{0} \tau_{0}\right) x_{0}^{\prime}\left(s-\omega_{0} \tau_{0}\right)\left(\omega_{1} \tau_{0}+\omega_{0} \tau_{1}\right) \\
& +\frac{1}{2} a_{1} x_{0}^{\prime \prime}\left(s-\omega_{0} \tau_{0}\right)\left(\omega_{1} \tau_{0}+\omega_{0} \tau_{1}\right)^{2}
\end{aligned}
$$

We take the solution of (49) as follows:

$$
x_{0}(s)=A_{0} \sin s+B_{0} \cos s,
$$

where $A_{0}$ and $B_{0}$ are constants. Next we substitute (52) into (49) and derive that $A_{0}$ and $B_{0}$ are arbitrary. Without loss of generality, we impose the initial conditions $x_{0}(0)=0$ and $x_{0}^{\prime}(0)=1$ and get from (52) that

$$
x_{0}(s)=\sin s .
$$

Next, we look for a solution to (50) as

$$
x_{1}(s)=A_{1} \sin s+B_{1} \cos s+C_{1} \sin (2 s)+D_{1} \cos (2 s)+E_{1},
$$

where the coefficients $A_{1}, B_{1}, C_{1}, D_{1}$, and $E_{1}$ are constants. Substituting (53) and (54) in (50) and equating the coefficients of the resonant terms $\sin s, \cos s, \sin (2 s)$, and $\cos (2 s)$, we find that

$$
\begin{gathered}
\omega_{1}=\tau_{1}=0, \quad C_{1}=\frac{M_{1} M_{3}+M_{2} M_{4}}{M_{1}^{2}+M_{2}^{2}}, \\
D_{1}=\frac{M_{2} M_{3}-M_{1} M_{4}}{M_{1}^{2}+M_{2}^{2}}, \quad E_{1}=-\frac{1+a_{2}}{2\left(a_{0}+a_{1}\right)},
\end{gathered}
$$

with $A_{1}$ and $B_{1}$ being arbitrary and

$$
\begin{gathered}
M_{1}=\frac{2 \omega_{0}\left(a_{1}-a_{0}\right)}{a_{1}}, \quad M_{2}=\frac{\left(a_{0}+a_{1}\right)\left(a_{1}-2 a_{0}\right)}{a_{1}}, \\
M_{3}=\frac{a_{2}\left(a_{1}^{2}-2 a_{0}^{2}\right)-a_{1}^{2}}{2 a_{1}^{2}}, \quad M_{4}=-\frac{\omega_{0} a_{0} a_{2}}{a_{1}} .
\end{gathered}
$$

For simplicity, we let $A_{1}=B_{1}=0$. Hence, (54) becomes

$$
x_{1}(s)=C_{1} \sin (2 s)+D_{1} \cos (2 s)+E_{1},
$$

where $C_{1}, D_{1}$, and $E_{1}$ are given in (55). Finally, let

$$
\begin{aligned}
x_{2}(s)= & A_{2} \sin s+B_{2} \cos s+C_{2} \sin (2 s) \\
& +D_{2} \cos (2 s)+E_{2} \sin (3 s)+F_{2} \cos (3 s)+G_{2}
\end{aligned}
$$

be the solution of (51), with $A_{2}, B_{2}, C_{2}, D_{2}, E_{2}, F_{2}$, and $G_{2}$ being constants. Using (53), (57), and (58) into (51), after trigonometric simplifications have been performed, we obtain

$$
\begin{aligned}
\left(\omega_{0} A_{2}+\omega_{2}\right) \cos s-\omega_{0} B_{2} \sin s+2 \omega_{0} C_{2} \cos (2 s) & -2 \omega_{0} D_{2} \sin (2 s)+3 \omega_{0} E_{2} \cos (3 s)-3 \omega_{0} F_{2} \sin (3 s) \\
= & {\left[\omega_{0}\left(\omega_{2} \tau_{0}+\omega_{0} \tau_{2}\right)-\omega_{0} B_{2}+N_{1}\right] \sin s } \\
+ & {\left[a_{0}\left(\omega_{2} \tau_{0}+\omega_{0} \tau_{2}\right)+\omega_{0} A_{2}+N_{2}\right] \cos s } \\
+ & {\left[a_{0} C_{2}+a_{1}\left(C_{2} N_{4}+D_{2} N_{3}\right)\right.} \\
& \left.\quad-a_{2}\left(A_{1} N_{3}-B_{1} N_{4}\right)\right] \sin (2 s) \\
+ & {\left[a_{0} D_{2}+a_{1}\left(-C_{2} N_{3}+D_{2} N_{4}\right)\right.} \\
& \left.\quad-a_{2}\left(A_{1} N_{4}+B_{1} N_{3}\right)\right] \cos (2 s) \\
+ & {\left[a_{0} E_{2}+a_{1}\left(E_{2} N_{5}+F_{2} N_{6}\right)\right] \sin (3 s) } \\
+ & {\left[a_{0} F_{2}+a_{1}\left(F_{2} N_{5}-E_{2} N_{6}\right)\right] \cos (3 s) } \\
+ & a_{0} G_{2}+a_{1} G_{2}+a_{2} A_{1},
\end{aligned}
$$

where

$$
\begin{gathered}
N_{1}=-\frac{2 E_{1} a_{0} a_{2}+C_{1} a_{2} \omega_{0}-D_{1} a_{0} a_{2}}{a_{1}}, \\
N_{2}=\frac{2 E_{1} a_{2} \omega_{0}-D_{1} a_{2} \omega_{0}-C_{1} a_{0} a_{2}}{a_{1}}, \quad N_{3}=\frac{2 a_{0} \omega_{0}}{a_{1}^{2}}, \\
N_{4}=\frac{2 a_{0}^{2}-a_{1}^{2}}{a_{1}^{2}}, \quad N_{5}=-\frac{4 a_{0}^{3}-3 a_{0} a_{1}^{2}}{a_{1}^{3}}, \\
N_{6}=-\frac{3 a_{1}^{2} \omega_{0}-4 \omega_{0}^{3}}{a_{1}^{3}} .
\end{gathered}
$$

Comparing the coefficients of the terms, $\sin s, \cos s, \sin (2 s)$, $\cos (2 s), \sin (3 s)$, and $\cos (2 s)$, we get the following expressions:

$$
\begin{aligned}
& \omega_{2}=\frac{N_{2} \omega_{0}-N_{1} a_{0}}{\omega_{0}}, \\
& \tau_{2}=\frac{N_{1}\left(a_{0} \tau_{0}-1\right)-N_{2} \omega_{0} \tau_{0}}{\omega_{0}^{2}} .
\end{aligned}
$$

Summing up all the above analysis, the bifurcated periodic solution of (4) has an approximation of the form

$$
x(s)=\sqrt{\frac{\tau-\tau_{0}}{\tau_{2}}} x_{0}(s)+\frac{\tau-\tau_{0}}{\tau_{2}} x_{1}(s)+\cdots,
$$

where $\tau \approx \tau_{0}+\tau_{2} \varepsilon^{2}, \omega \approx \omega_{0}+\omega_{2} \varepsilon^{2}$, with $x_{0}(s)$ and $x_{1}(s)$ given in (53) and (57), respectively. Here, the parameters $\tau_{2}$ and $\omega_{2}$ determine the direction of the Hopf bifurcation and the period of the bifurcating periodic solution, respectively. We have the following result. 
Theorem 2. The Hopf bifurcation of (1) at the equilibrium point $k_{*}$ when $\tau=\tau_{*}$ is supercritical (resp., subcritical), if $\tau_{2}>$ 0 (resp., $\left.\tau_{2}<0\right)$ and the bifurcating periodic solutions exist for $\tau>\tau_{*}$ (resp., $\tau<\tau_{*}$ ). In addition, its period decrease (resp., increases) as $\tau$ increases, if $\omega_{2}>0\left(\right.$ resp., $\left.\omega_{2}<0\right)$.

Remark 3. Let $\beta=4 \alpha$ and $\alpha=1$. Then

$$
\begin{gathered}
M_{2}=0, \quad C_{1}=M_{3}=-\frac{1}{16}, \\
D_{1}=-M_{4}=-2 \sqrt{3}, \quad E_{1}=-\frac{1}{2}, \quad \tau_{0}=\frac{2 \pi}{3 \sqrt{3}} .
\end{gathered}
$$

As direct calculation shows that (61) yields $\omega_{2}>0$ and $\tau_{2}<$ 0 . In this case, the Hopf bifurcation is subcritical and the bifurcating periodic solutions exist for $\tau<\tau_{*}$. Moreover, its period decreases as $\tau$ increases.

\section{Conclusions}

In this paper, we consider the special neoclassical growth model with fixed time delay introduced and examined by Matsumoto and Szidarovszky's [5], where a mound-shaped production function for capital growth was assumed in the dynamic equation. In their model, the stability can be lost at a certain value of the delay and the equilibrium remains unstable afterwards. At this critical value, Hopf bifurcation occurs. By applying the normal form theory and the center manifold theorem, we derive explicit formulae which determine the stability and direction of the bifurcating periodic solutions. Moreover, we employ Lindstedt's perturbation theory to approximate the bifurcated periodic solution and provide approximate expressions for the amplitude and frequency of the resulting limit cycle as a function of the model parameters.

\section{Conflict of Interests}

The authors declare that there is no conflict of interests.

\section{Acknowledgment}

The authors would like to thank the referees for their valuable comments.

\section{References}

[1] P. K. Asea and P. J. Zak, "Time-to-build and cycles," Journal of Economic Dynamics \& Control, vol. 23, no. 8, pp. 1155-1175, 1999.

[2] P. J. Zak, "Kaleckian lags in general equilibrium," Review of Political Economy, vol. 11, pp. 321-330, 1999.

[3] M. Szydłowski, "Time to build in dynamics of economic models. II. Models of economic growth," Chaos, Solitons and Fractals, vol. 18, no. 2, pp. 355-364, 2003.

[4] M. Szydłowski and A. Krawiec, "A note on Kaleckian lags in the Solow model," Review of Political Economy, vol. 16, no. 4, pp. 501-506, 2004.

[5] A. Matsumoto and F. Szidarovszky, "Delay differential neoclassical growth model," Journal of Economic Behavior and Organization, vol. 78, no. 3, pp. 272-289, 2011.
[6] A. Matsumoto, F. Szidarovszky, and H. Yoshida, "Dynamics in linear cournot duopolies with two time delays," Computational Economics, vol. 38, no. 3, pp. 311-327, 2011.

[7] H. d'Albis, E. Augeraud-Veron, and A. Venditti, "Business cycle fluctuations and learning-by-doing externalities in a one-sector model," Journal of Mathematical Economics, vol. 48, no. 5, pp. 295-308, 2012.

[8] M. Bambi, G. Fabbri, and F. Gozzi, "Optimal policy and consumption smoothing effects in the time-to-build AK model," Economic Theory, vol. 50, no. 3, pp. 635-669, 2012.

[9] R. Boucekkine, G. Fabbri, and P. Pintus, "On the optimal control of a linear neutral differential equation arising in economics," Optimal Control Applications \& Methods, vol. 33, no. 5, pp. 511530, 2012.

[10] A. Matsumoto and F. Szidarovszky, "Nonlinear delay monopoly with bounded rationality," Chaos, Solitons and Fractals, vol. 45, no. 4, pp. 507-519, 2012.

[11] L. V. Ballestra, L. Guerrini, and G. Pacelli, "Stability switches and Hopf bifurcation in a Kaleckian model of business cycle," Abstract and Applied Analysis, vol. 2013, Article ID 689372, 8 pages, 2013.

[12] C. Bianca and L. Guerrini, "On the Dalgaard-Strulik model with logistic population growth rate and delayed-carrying capacity," Acta Applicandae Mathematicae, vol. 128, pp. 39-48, 2013.

[13] C. Bianca, M. Ferrara, and L. Guerrini, "Stability and bifurcation analysis in a Solow growth model with two time delays," Abstract and Applied Analysis. In press.

[14] L. Guerrini and M. Sodini, "Nonlinear dynamics in the Solow model with bounded population growth and time-to-build technology," Abstract and Applied Analysis, vol. 2013, Article ID 836537, 6 pages, 2013.

[15] L. Guerrini and M. Sodini, "Dynamic properties of the Solow model with increasing or decreasing population and time-tobuild technology," Abstract and Applied Analysis. In press.

[16] A. Matsumoto and F. Szidarovszky, "Asymptotic behavior of a delay differential neoclassical growth model," Sustainability, vol. 5, no. 2, pp. 440-455, 2013.

[17] R. Day, "Irregular growth cycles," American Economic Review, vol. 72, pp. 406-414, 1982.

[18] B. D. Hassard, N. D. Kazarinoff, and Y. H. Wan, Theory and Applications of Hopf Bifurcation, vol. 41, Cambridge University Press, Cambridge, Mass, USA, 1981.

[19] A. H. Nayfeh, Introduction to Perturbation Techniques, John Wiley \& Sons, New York, NY, USA, 1981.

[20] N. MacDonald, Time Lags in Biological Models, vol. 27 of Lecture Notes in Biomathematics, Springer, Berlin, Germany, 1978. 


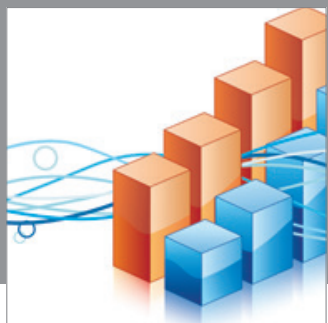

Advances in

Operations Research

mansans

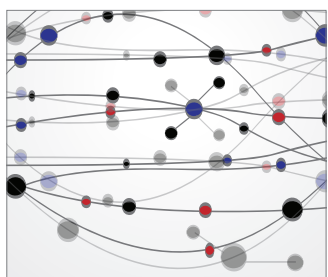

The Scientific World Journal
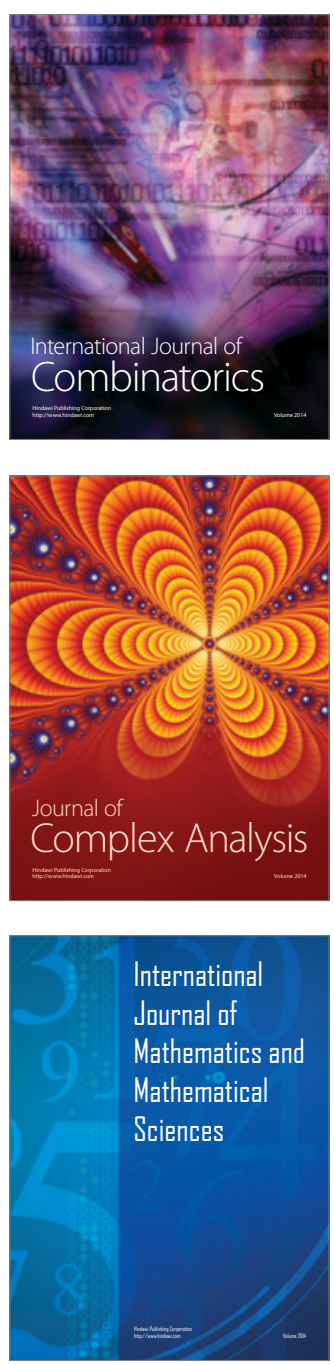
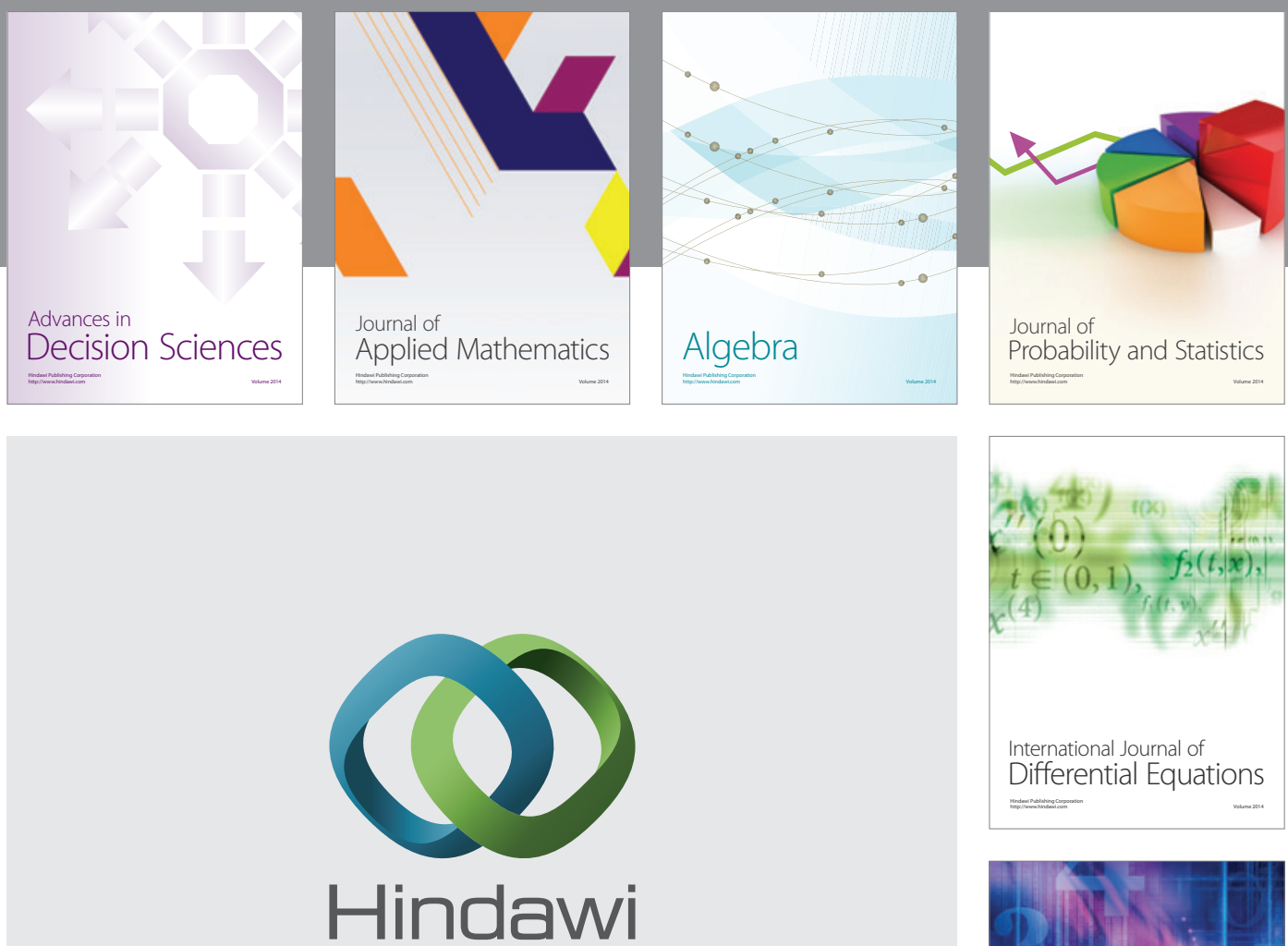

Submit your manuscripts at http://www.hindawi.com
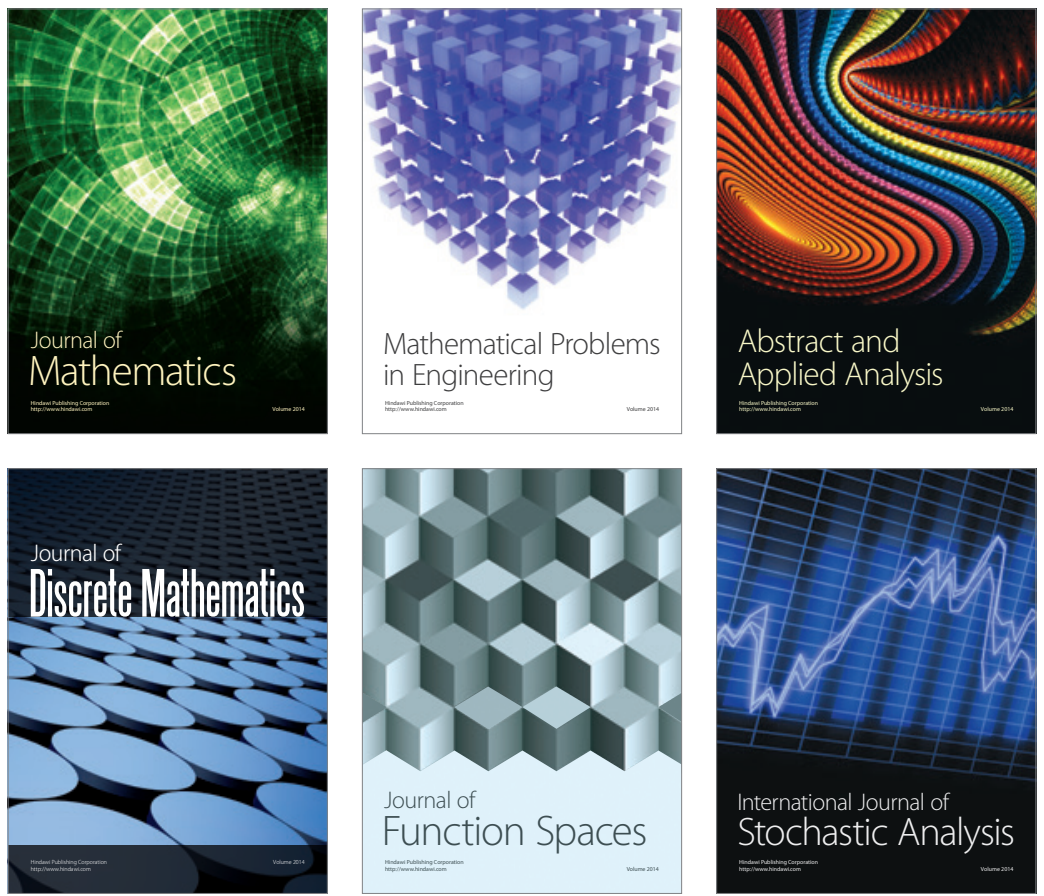

Journal of

Function Spaces

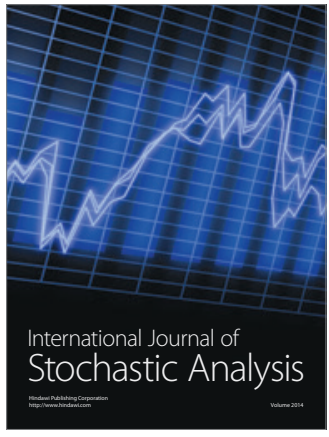

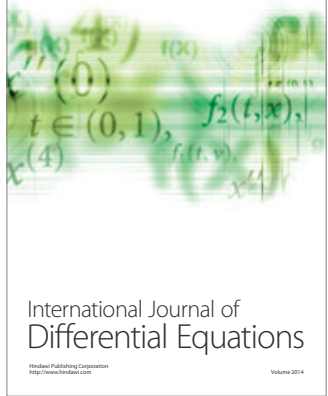
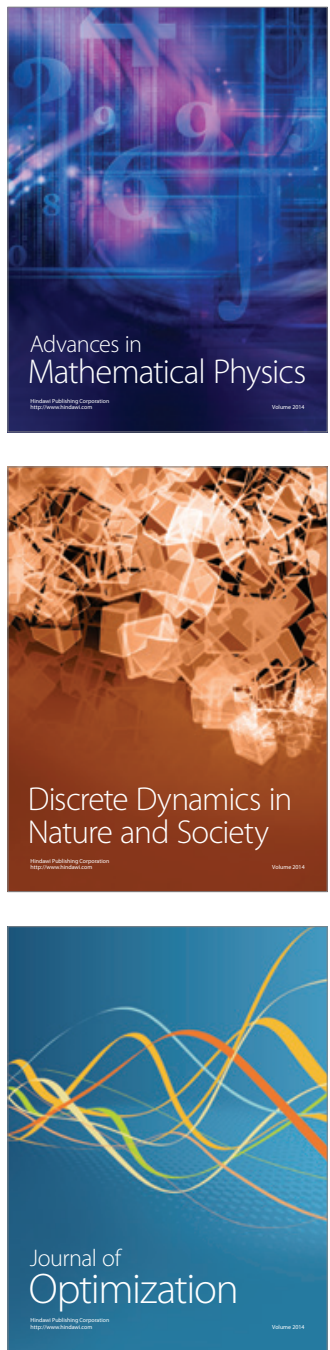